\title{
Does perceptual or motor experience influence the perception of global and joint-specific kinematic changes in complex movement patterns?
}

\author{
Georgia Giblin $^{1,2,4}$ - Damian Farrow ${ }^{3,4} \cdot$ Machar Reid $^{2,5}$ - Kevin Ball ${ }^{4}$. \\ Bruce Abernethy ${ }^{6}$
}

Published online: 23 June 2016

(C) The Psychonomic Society, Inc. 2016

\begin{abstract}
The perception and identification of technical errors during skill execution is a critical component in coaching, because it provides the foundation for skill analysis, instruction, and feedback provision. In this study, we examined the influences of perceptual and motor experience on the perception of kinematic change in a technical evaluation task that is common in coaching. A total of 21 expert coaches, stratified by playing ("motor") expertise, as well as ten novice coaches and ten current players, observed video and point-light displays of a service action and recorded written judgments on whether the serve had changed from one video clip to the next. Three kinematic variables were manipulated: maximum knee flexion, maximum trunk rotation, and ball toss position at zenith. Coaching experience provided no additional benefit when perceiving global (holistic) changes in the service action, and limited if any benefit when perceiving specific changes in the observed kinematics. A significant
\end{abstract}

Georgia Giblin

Georgia.Giblin7@gmail.com

1 Queensland Academy of Sport, Brisbane, Queensland, Australia

2 Insight and Innovation, Tennis Australia, Melbourne, Victoria, Australia

3 Australian Institute of Sport, Canberra, Australia

4 ISEAL, Victoria University, Melbourne, Victoria, Australia

5 School of Sport Science, Exercise and Health, University of Western Australia, Perth, Western Australia

6 School of Human Movement \& Nutrition Sciences, University of Queensland, Brisbane, Queensland, Australia expertise effect showed that expert coaches have increased sensitivity when detecting smaller changes in knee flexion. Changes in trunk rotation appeared difficult to perceive for all groups, whereas changes in lateral ball toss position were easily perceived. Motor experience did not influence the perception of global kinematics and provided no benefit above perceptual experience alone when perceiving specific kinematic changes. Collectively, the findings demonstrate that experienced coaches have the capacity to detect relatively small changes in kinematics; however, the ability to detect changes does not appear to be influenced by the extent of their own expertise in producing the movement pattern being evaluated.

Keywords Perception $\cdot$ Kinematics $\cdot$ Coaching $\cdot$ Motor expertise $\cdot$ Error detection

The ability to accurately perceive movement patterns is a critical skill that many observers spend countless hours refining. Highly refined visual perceptual skill is particularly important in sports, where coaches are continually observing and evaluating the movement patterns of their athletes to provide feedback and facilitate skill (re)acquisition. However, despite the obvious importance of visual observation and error detection in coaching, the assessment of the capacities of coaches of different levels of experience to detect changes in the kinematics of complex movement patterns remains relatively unexplored.

An emerging body of research has attempted to understand expertise in coaching and how it may be developed (Côté \& Gilbert, 2009; Ford, Coughlan, \& Williams, 2009; Wiman, Salmoni, \& Hall, 2010). Although the expert performance approach has demonstrated that expert performers hold some clear advantages over their less-skilled counterparts when 
performing, the literature surrounding the locus of coaching expertise is less definitive. Nevertheless, a few key characteristics (e.g., perceptual-cognitive factors) seem to consistently underpin expertise in coaching and are not dissimilar from those that differentiate experts from less-skilled performers. Sherman, Sparrow, Jolley, and Eldering (2001) investigated the perceptual skill of expert and novice golf coaches by examining their abilities to predict a golfer's handicap on the basis of internal models of golf biomechanics, and concluded that the expert and novice coaches had similar abilities to identify the fundamental characteristics of the swing, although a limitation in this study was the possible disruption of critical relative motion cues. Relative motion has been shown to be a key factor in the perception of action (Abernethy \& Russell, 1987), and disruption to the normal motion of the swing may well have affected the ways that the coaches perceived the action. Although Sherman et al. (2001) compared differences between coaches in their abilities to perceive kinematics, they did not examine the coaches' abilities to perceive change. Given that one of the major aims of coaching is to provide corrective feedback in order to facilitate improvement, the ability to distinguish key kinematic changes from one execution of a skill to the next is crucial and has not yet been a focus of systematic examination.

Given that in many sports the coaches are past performers of the activity for which they now coach, it is of interest to ascertain whether the perceptual-cognitive skills of coaches are, in any way, related to the extent of their experience and expertise in physically performing the skills in which they now instruct others. Common-coding theory (Prinz, 1997; Schütz-Bosbach \& Prinz, 2007) suggests that observers should be more perceptually attuned to actions that they have had experience performing, and this view is supported by neural evidence of mirror neurons that activate when observing or producing a specific action (Buccino, Binkofski, \& Riggio, 2004), and by functional magnetic resonance imaging (fMRI) results indicating activation of the mirror neuron system in both action perception and production (Calvo-Merino, Glaser, Grèzes, Passingham, \& Haggard, 2006). On this basis, it is possible that coaches with a previous playing background (i.e., with motor experience) may have an advantage over those who do not when it comes to perceptually discriminating changes in movement patterns. In sports judging, a task with some similar requirements to coaching, specific motor experience has been shown to facilitate the perception of joint angles when judging a balance beam skill in gymnastics (Pizzera, 2012). More recently, Gründel, Schorer, Strauss, and Baker (2013) found similarities in the perceptual-cognitive skills used by coaches and players, suggesting there might be some transfer of the specific perceptual-cognitive skills developed as players to those required for coaching. Although research has identified some perceptual-cognitive skills that appear to transfer from playing to coaching, or from performing to judging, the evidence on motor expertise is far from compelling, and further research will be needed to determine whether, or how, motor expertise may influence the perception of movement kinematics.

The skill of sports coaches in discriminating changes in athlete technique has received little attention in the literature to date; however, the capacity of expert observers to perceive movement and discriminate change has received some attention in a clinical setting. Physical therapists commonly use visual observation to evaluate movement, with a particular focus on identifying movement deficiencies in order to formulate a treatment plan. Research using a just noticeable difference (JND) paradigm from psychophysics (i.e., the smallest detectable difference between two stimuli that is judged correctly $50 \%$ of the time; Kantowitz, Roediger, \& Elmes, 2009) has shown that observers have the capacity to detect changes as small as $2^{\circ}$ in trunk posture, with novice observers recording a larger JND (lower sensitivity) than expert observers (Weir et al., 2007). Although such research suggests quite sensitive discrimination of movement by experienced observers, the environment in which these judgments occur is often highly controlled and not necessarily reflective of the situation faced by other skill acquisition facilitators, especially sports coaches, who must evaluate movements executed at high speed.

In contrast to the existing evidence on coaching expertise, a sustained research program focused on expert performers of movement skills has led to a rich understanding of the perceptual-cognitive skills that underpin skilled performance. Existing evidence suggests that skilled performers, in contrast to novices, can more effectively and efficiently predict a movement's outcome from an opposition player on the basis of advance kinematic information (Abernethy \& Zawi, 2007; Farrow \& Abernethy, 2003). For example, skilled tennis players are superior to their less-skilled counterparts in anticipating serve direction when vision is occluded at or $300 \mathrm{~ms}$ prior to racket-ball contact (Farrow, Abernethy, \& Jackson, 2005). Experts appear to be sensitive to all of the same kinematic cues as nonexperts, as well as to some early-occurring sources of information to which the novices are not attuned (Abernethy \& Russell, 1987). For example, Müller et al. (2006) showed that expert cricket batsmen were able to use information from across linked body segments to successfully anticipate the intended delivery type and length, whereas the less-skilled players could not. The accurate perception of advance information by skilled performers affords them more time to initiate and perform interceptive actions. The expert players' advantage in the perception of movement patterns appears to be a consequence of their sensitivity to basic biomechanical/kinematic information in their opponent's movement pattern - a notion supported by preservation of the expert advantage when movement pattern information is presented simply through point-light displays (PLDs) of the 
motion of key joint segments (e.g., Abernethy, Gill, Parks, \& Packer, 2001).

The purpose of this study was to examine the influences of coaching (perceptual) and playing (motor) expertise on the capability to detect changes in a natural movement patternspecifically, the tennis serve. A sample of expert coaches (perceptual experts), novice coaches (perceptual novices), expert coaches with a past playing history (perceptual and motor experts), and current players (perceptual novices in terms of coaching [error detection] and motor experts) performed two discrimination tasks in which their perceptual sensitivity to kinematic changes was measured. With a growing body of research suggesting that motor expertise provides an advantage on perceptual tasks involving the same movement patterns (Aglioti, Cesari, Romani, \& Urgesi, 2008; Pizzera, 2012), we also sought to test whether this holds true in kinematic error detection tasks of the type frequently required in coaching. Given that previous work has demonstrated successful information pick-up from kinematics alone (e.g., Abernethy \& Zawi, 2007), each discrimination task was presented in both video and PLD formats, to establish whether the perception of kinematic change was based on the kinematics of the action or on other, nonkinematic sources of information. PLDs only provide kinematic information, whereas video displays provide the kinematic information plus other potential sources of information, such as textual, color, and context cues. We hypothesized that expert coaches would show a superior sensitivity to detecting both global and local changes in movement patterns, and that motor experience would provide an added benefit above perceptual experience alone, allowing the expert coaches with motor expertise to outperform those without. We also predicted that the advantages for the expert coaches would hold across both PLD and video presentation modes, indicating that kinematic information pick-up is fundamental to the accurate perception of movement pattern changes. We anticipated that players would be less sensitive to detecting changes than expert coaches, but would perform similarly to novice coaches, on account of their perceptual experience gained from playing.

\section{Method}

\section{Participants}

A total of 41 participants provided informed consent and completed the experiment. They consisted of eleven (all male) expert coaches (EC1; $M_{\mathrm{age}}=35.45$, age range 29-45 years), ten (eight males and two females) novice coaches (NC; $M_{\text {age }}=$ $25.6 \pm 2$, age range $24-30$ years), ten (nine males and one female) expert coaches with a previous high-level playing background (EC2; $M_{\text {age }}=40.5$, age range $30-57$ years), and ten (eight males and two females) players (PL; $M_{\text {age }}=17.3$, age range 16-19 years) without coaching experience (see Table 1). The participants were assigned to groups based on their coaching experience, level of accreditation obtained from their national governing body of tennis, and playing expertise as determined by their international playing rank. The expert coaches held (or were enrolled in the course to acquire, at the time of testing) the national governing body's highperformance coaching accreditation - the highest level of accreditation offered - which prepares coaches to work with nationally and internationally ranked professional players. The EC1 group had $15.2 \pm 4.7$ years of coaching experience, exceeding the 10 years frequently suggested as being necessary to become an expert (Ericsson, Krampe, \& Tesch-Römer, 1993). Although the members of the EC1 group were likely to have a high degree of motor familiarity with the serve, they were not considered motor experts given their ranking history as a player. The novice group had $5.9 \pm 2.3$ years of coaching experience, and each member held a level-one accreditation, which focuses on the development of junior players. The EC2 coaches (coaching experience $=11.25 \pm 8$ years) also held the national governing body's high-performance accreditation and had attained a high level of playing expertise by achieving a ranking inside the Association of Tennis Professionals top 500 during their playing career. As such, the EC2 group were considered both perceptual and motor experts. The PL group consisted of current nationally ranked junior players with no coaching experience. The PL group was considered to have a degree of perceptual experience similar to that among the NC group, given their degree of experience with the serve gained

Table 1 Coaching and playing experience histories of participants

\begin{tabular}{lcllll}
\hline Group & Age (years) & $\begin{array}{l}\text { Coaching } \\
\text { Experience (years) }\end{array}$ & $\begin{array}{l}\text { Coaching } \\
\text { Accreditation Level }\end{array}$ & $\begin{array}{l}\text { Level of Player Coached } \\
\text { Highest } \\
\text { Playing Rank }\end{array}$ \\
\hline NC & $25.6 \pm 2.07$ & $5.9 \pm 2.3$ & 1 & Beginner-intermediate juniors & N/A \\
EC1 & $35.45 \pm 5.1$ & $15.2 \pm 4.7$ & 3 & ITF juniors and professional ATP players & N/A \\
EC2 & $40.5 \pm 6.7$ & $11.25 \pm 8.0$ & 3 & ITF juniors and professional ATP players & $150 \pm 176.1$ \\
PL & $17.1 \pm 1.1$ & 0 & N/A & None & $17 \pm 5.6$ \\
\hline
\end{tabular}


through playing rather than coaching, but to possess superior motor expertise. The research was approved by the university's human research ethics committee.

\section{Construction of test video}

One skilled male player ( 24 years of age), currently ranked inside the national top 100 and competing at the highest level of competition within his state, was recruited to perform the tennis serves that provided the basis for the test stimuli for this experiment. These serves were simultaneously recorded on digital video and by motion capture. The participant was fitted with 80 retro-reflective 14-mm markers in the University of Western Australia's full-body marker set (Lloyd, Alderson, \& Elliott, 2000; Reid, Whiteside, Giblin, \& Elliott, 2013). Once all markers had been fitted to the participant, they were securely fastened to the skin using athletic tape. In addition, three markers were affixed to the racket, and three ultralight hemispherical markers were attached to the ball. After attaching all markers, the participant undertook a 15-min dynamic warm-up similar to one he would perform before training or a game. After warming up, the player was given sufficient time to familiarize himself with the testing environment and to hit a number of practice serves until he felt ready to begin serving with maximal effort.

The server was filmed as he hit a total of ten successful flat first serves with maximal effort to a target area $(1.2 \mathrm{~m}$ in width $\times 1 \mathrm{~m}$ in length), bordering the intersection of the service line and center service line of the court. These trials were used to establish the server's usual service technique. The target area was affixed to the ground using white tape to ensure that the target was visible to the player. A serve was considered successful if it landed within this target area. The server was given as many serves as were required to hit ten successful regular first serves. Following this, the server followed a script (see Table 2) that was intended to elicit changes in the kinematics of knee flexion, trunk rotation, and ball toss position, respectively, relative to those used in the standard serve. The server was required to hit five successful serves under each instructional set. The aforementioned three kinematics were manipulated because of their importance to successful serve technique (Elliott, Reid, \& Crespo, 2003).

\section{Materials}

A ten-camera, 500-Hz VICON MX optical motion analysis system (Oxford Metrics Inc, UK) was used to capture all marker trajectories and calculate the resulting service kinematics. Gaps in the marker trajectories were filled using the cubic spline interpolation function within VICON Nexus. A Woltring filter with an optimal mean squared error of $3 \mathrm{~mm}$ as determined by a residual analysis was applied to the raw data, which were modeled using the University of Western Australia's customized model to calculate body kinematics (Lloyd et al., 2000; Whiteside, Chin, \& Middleton, 2013). In addition, two high-definition JVC (ProHD GY-HM100E, Japan) digital video cameras were used to capture the service action. One camera was positioned $5 \mathrm{~m}$ directly behind the service tee $(\mathrm{T})$, perpendicular to the baseline, to capture the serve from a rear perspective. The second digital camera was placed parallel to the baseline $2 \mathrm{~m}$ from the sideline, to capture a sagittal or side view of the serve.

An average representation of the player's normal serve was determined from the initial ten trials and used as a reference to which all other serves were compared. The kinematic changes elicited by the instructions were analyzed, and a series of incremental changes were used to create the paired clips that would be seen by the participant groups (see Table 3).

To examine whether change discrimination was based purely on the perceivable kinematics, a PLD condition was created in addition to a standard two-dimensional video condition. The PLD clips showed only the pure kinematics of the serve and were created from the 3-D data that were collected. Following reconstruction of the 3-D marker trajectories in VICON Nexus, the c3d file was imported into the Visual 3D software (C-Motion Inc., Germantown, MD), where any unnecessary markers were deleted. A total of 17 marker trajectories were used to create the PLD of the serve (see Fig. 1); these included the markers on the left and right lateral malleolus, lateral epicondyles of the knee, anterior superior iliac spine, acromion process, lateral epicondyle of the elbow, and ulnar styloid process (wrist), as well as one on the head. One marker was also used to represent the ball origin, plus three markers to represent the racket.

Test videos were created for two experimental task conditions - a global discrimination task and a specific kinematic discrimination task. Each condition consisted of both video and PLD viewing conditions, in which the test clips were identical in nature. Each test video consisted of 30 paired video clips. The first clip in the pair was always the same reference serve, to which the following serve was to be compared. The second clip differed in the amount of knee flexion, the amount of trunk rotation, or the ball toss positioning, as compared to the first (reference) clip (see Table 3).

Two test videos were created for the global task. In this condition, the second clip was either identical to the first or differed in any of the three kinematic variables by a moderate amount $\left(\sim 4.5^{\circ}\right)$. A total of 15 clips were presented in which the comparison serves in the pair were the "same," and 15 clips in which the serves were "different." For the specific discrimination task, in which a change in only one of the designated kinematic variable had to be judged, a total of six test videos were created (3 kinematic variables $\times 2$ viewing conditions). Six incremental levels of change were presented five times each in a randomized order (see Fig. 2). An intertrial 
Table 2 Instructions given to the model in creation of the comparative stimuli

\begin{tabular}{ll}
\hline Variable & Instruction \\
\hline Knee Flexion & Using your normal serve as a reference, perform \\
& ten serves with more knee flexion \\
& Using your normal serve as a reference, perform \\
& ten serves with less knee flexion \\
Trunk Rotation & Using your normal serve as a reference, perform \\
& ten serves with more trunk rotation \\
& Using your normal serve as a reference, perform \\
& ten serves with less trunk rotation \\
& Using your normal serve as a reference, perform \\
& ten serves moving the ball at its peak $10 \mathrm{~cm}$ to \\
Ball Toss & your right \\
& Using your normal serve as a reference, perform \\
& ten serves moving the ball at its peak $10 \mathrm{~cm}$ \\
to your left
\end{tabular}

interval of $5 \mathrm{~s}$ between pairs allowed the participants sufficient time to make their judgments in both tasks.

\section{Procedure and design}

In the global condition, a two-choice discrimination task required the participants to judge whether the service action in the second clip in the pair was the "same" as or "different" from the serve in the first. In the specific condition, a threechoice discrimination task required participants to detail how a specific kinematic variable within the service action had changed. Three kinematic variables of interest were manipulated: maximum knee flexion, maximum trunk rotation, and ball toss positioning at its peak. Changes were to be judged as either "more," "less," or the "same" in the knee and trunk conditions, or "right," "left," or the "same" in the ball condition, with the change, or lack of it, referenced to the standard clip shown first.

Participants were seated $3 \mathrm{~m}$ from the video image $(3 \mathrm{~m}$ high and $1.6 \mathrm{~m}$ wide), which was projected onto a large wall directly in front of the participants. The participants were instructed that they would be required to judge the difference between two serves presented as a pair. They were informed that the first serve would always be the same, and that the second might be different. In the specific discrimination task, their attention was directed to the specific variable that was changing. For example, they were instructed to "view the ball position at the top of the ball toss." Each participant was given a response sheet and was required to circle their response indicating how the second clip differed from the first, and to rate their confidence in their judgments on a 6-point Likert scale, with $50 \%$ representing a guess, and $100 \%$ representing certainty. Two familiarization trials preceded each test video. The test videos were always presented so that the global discrimination task was presented first, followed by the specific discrimination task. Viewing condition (video or PLD) presentation was counterbalanced across groups.

\section{Data analysis}

Global discrimination task Responses in the global condition were analyzed using signal detection theory (SDT), to measure both the sensitivity of the observer to perceiving change and any associated response bias. Correctly detecting the signal when present ("different" trials) was considered a hit, whereas incorrectly reporting a pair of trials as different when they were actually the same was considered a false alarm. Sensitivity $\left(d^{\prime}\right)$ and the criterion $(c)$ were calculated according to equation 9.8 of Macmillan and Creelman (2004). Confidence was assessed using a 4 (Group) $\times 2$ $($ View $) \times 2$ (Same, Different) analysis of variance.

Specific discrimination task Performance on the specific discrimination task was assessed in terms of response accuracy (RA). Given the dynamic nature of the tennis serve and its degrees of freedom, SDT was not suitable to analyze accuracy in the specific discrimination task. Rather, in this task coaches were awarded one point per correct judgment. Each level of change was viewed a total of five times, so a maximum of five points (100\% RA) were available for each level of change within each viewing perspective. RA significantly less than $33 \%$ was considered to represent guessing (since there were

Table 3 Test pairs created and used in the experiment proper

\begin{tabular}{lllr}
\hline Level of Change & Knee Flexion & Trunk Rotation & Ball Zenith Position \\
\hline L1 & $\begin{array}{c}\text { Reference-reference } \\
\text { (no change) } \\
\text { Reference }+1.6^{\circ}\end{array}$ & $\begin{array}{c}\text { Reference-reference } \\
\text { (no change) } \\
\text { Reference }-1.6^{\circ}\end{array}$ & $\begin{array}{c}\text { Reference-reference } \\
\text { (no change) }\end{array}$ \\
L2 & Reference $-3.3^{\circ}$ & Reference $-2.75 \mathrm{~cm}$ \\
L3 & Reference $+4.7^{\circ}$ & Reference $-4.6^{\circ}$ & Reference $-5.3 \mathrm{~cm}$ \\
L4 & Reference $-5.8^{\circ}$ & Reference $+6.4^{\circ}$ & Reference $-7.5 \mathrm{~cm}$ \\
L5 & Reference $+7.5^{\circ}$ & Reference $-8.7^{\circ}$ & Reference $-11 \mathrm{~cm}$ \\
L6 & & Reference $-14 \mathrm{~cm}$ \\
\hline
\end{tabular}




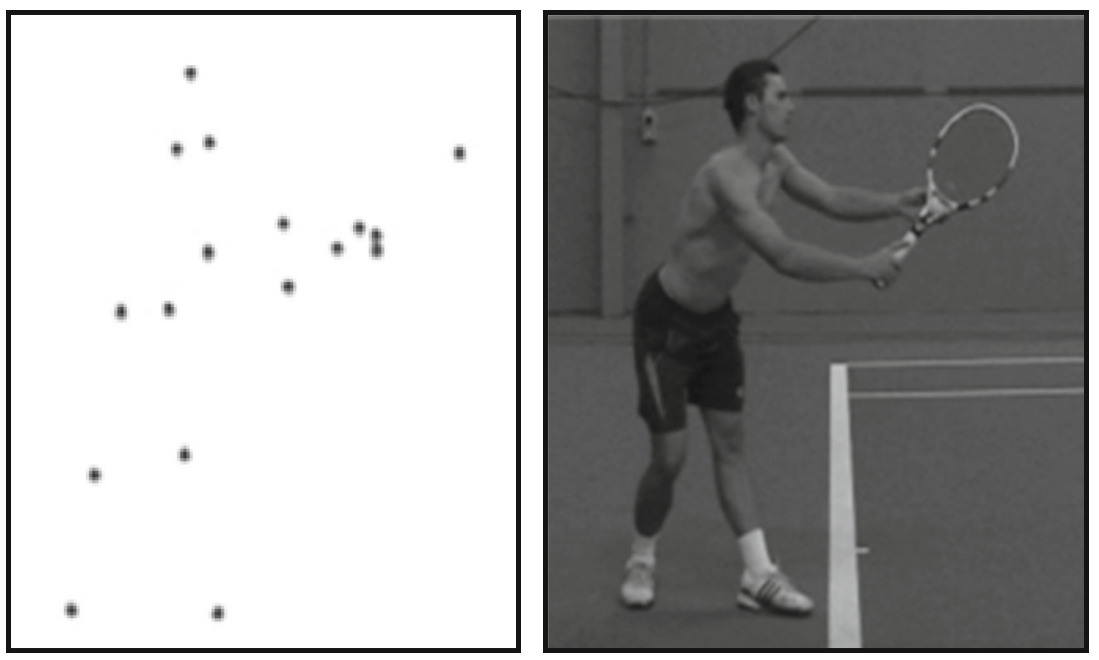

Fig. 1 A static example of the point-light display using 17 markers (dots) and the comparable video frame

three options per judgment). Three separate fully factorial analyses of covariance were used to assess the influences of coaching experience (NC, EC1, EC2, PL), level of change (16), and viewing perspective (video or PLD) on RA. Coaching experience was a between-groups variable, age was used as a covariate, and viewing condition and level of change were within-subjects variables. Alpha was set at .05 for all statistical tests. Significant three-way interactions between expertise, viewing condition, and level of change, when obtained, were followed up by testing the two-way interaction between group and level of change for each view. A Bonferroni adjustment was applied in the event of multiple test applications. Greenhouse-Geisser adjustments to the degrees of freedom were applied in the event that the sphericity assumption was violated. Effect sizes $\left(\eta_{\mathrm{p}}{ }^{2}\right)$ are also reported where $F>1$. Spearman's rank correlation was used to assess the relationship between confidence and RA.

\section{Results}

\section{Global discrimination task}

The SDT results for the global discrimination task are presented in Table 4. Despite differences in the expertise levels of participants, all groups performed similarity when asked to judge global changes in the serve, as evidenced by similar hit rates. Hit rates decreased regardless of expertise when viewing the PLD, highlighting the increased difficulty faced by participants when judging change solely on the basis of kinematics. False alarm rates in the video condition were highest amongst NCs, suggesting that they were most likely to report the stimuli as different when they were in fact the same. Higher false alarm rates were evident in the PLD viewing condition, suggesting that coaches were more likely to report the stimuli as different when viewing the PLD.
Sensitivity $\left(d^{\prime}\right)$ significantly decreased when viewing the PLD, whereas the criterion (c) significantly increased. Participants were less sensitive to differences in the stimuli when viewing the PLD condition, in addition to being less likely to report the stimuli as different.

For confidence, a significant three-way interaction between coaching expertise, viewing perspective, and level of change was evident, $F(3,37)=4.252, p<.011, \eta^{2}=.256$. The threeway interaction was followed up by analyzing the twoway interaction between expertise and level of change for each viewing perspective. A significant two-way interaction between expertise and level of change, $F(3$, $37)=3.217, p=.034, \eta^{2}=.207$, was present in the video viewing condition. Follow-up analyses revealed no significant difference in confidence across expertise levels when viewing either trials that were the same or trials that were different, $F<1$. No higher-order interactions were evident in the PLD viewing condition, $F<1$. A main effect of viewing perspective revealed that confidence was significantly decreased for all coaches when viewing the PLDs, regardless of the level of change, $F(1$, 37) $=9.652, p=.004, \eta^{2}=.207$.

\section{Specific discrimination task}

Knee flexion We observed a significant effect of expertise on RA after controlling for the effect of age, $F(3$, $36)=3.549, p=.024, \eta_{\mathrm{p}}{ }^{2}=.028$ (see Figs. 3 and 4). A complex three-way interaction between coaching expertise, level of change, and viewing condition, $F(15,185)=1.801$, $p=.037, \eta_{\mathrm{p}}{ }^{2}=.127$, was evident. The significant three-way interaction was followed up by testing the interaction between expertise and level of change for each viewing condition, in order to answer our specific hypotheses. A significant main effect of expertise, $F(3,37)=4.834, p=.006, \eta_{p}{ }^{2}=.281$, revealed that expert coaches significantly outperformed both 


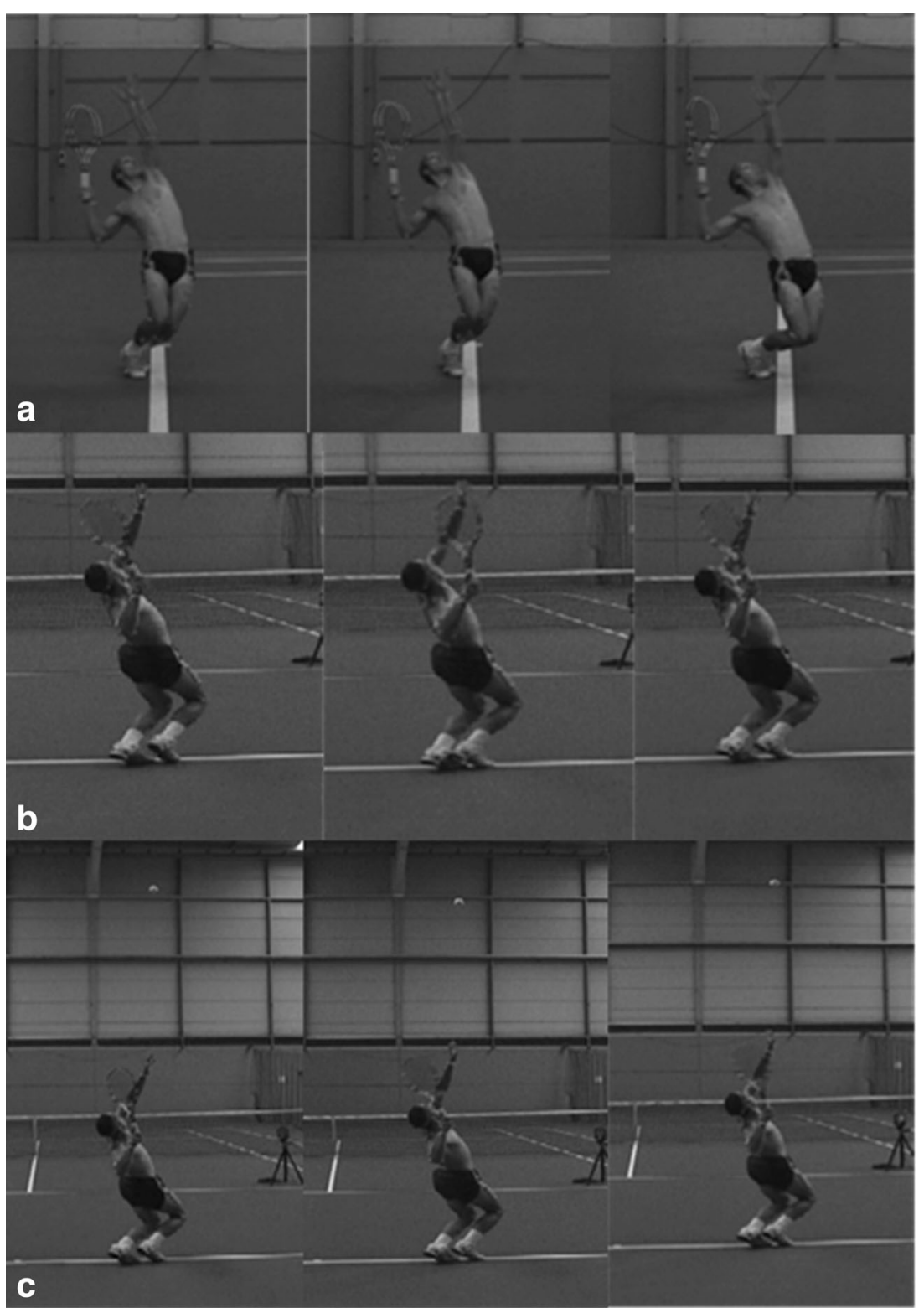

Fig. 2 Example screenshot of the reference (left), smallest change (middle), and largest change (right) serves presented to the participants for (a) maximum knee flexion, (b) maximum trunk rotation, and (c) ball toss positioning at zenith

Table 4 Signal detection theory results and confidence ratings for all groups on the global discrimination task

\begin{tabular}{|c|c|c|c|c|c|c|c|}
\hline Group & Viewing Condition & Hit Rate & False Alarm Rate & $d^{\prime}$ & $c$ & Confidence (Different) & Confidence (Same) \\
\hline \multirow[t]{2}{*}{$\mathrm{NC}$} & Video & $.71 \pm .14$ & $.27 \pm .15$ & $1.32 \pm 0.72$ & $0.05 \pm 0.32$ & $76.93 \pm 7.93$ & $72.2 \pm 6.75$ \\
\hline & PLD & $.43 \pm .12$ & $.21 \pm .18$ & $0.45 \pm 0.45$ & $0.40 \pm 0.31$ & $69.73 \pm 9.93$ & $70.1 \pm 13.00$ \\
\hline \multirow[t]{2}{*}{ EC1 } & Video & $.68 \pm .14$ & $.17 \pm .14$ & $1.45 \pm 0.68$ & $0.24 \pm 0.30$ & $86.26 \pm 7.40$ & $85.6 \pm 10.37$ \\
\hline & PLD & $.52 \pm .20$ & $.28 \pm .17$ & $0.74 \pm 0.70$ & $0.27 \pm 0.46$ & $77.70 \pm 9.62$ & $78.67 \pm 9.19$ \\
\hline \multirow[t]{2}{*}{ EC2 } & Video & $.73 \pm .17$ & $.21 \pm .15$ & $1.58 \pm 0.77$ & $0.12 \pm 0.35$ & $80.93 \pm 15.1$ & $79.27 \pm 15.20$ \\
\hline & PLD & $.51 \pm .22$ & $.35 \pm .11$ & $0.44 \pm 0.64$ & $0.20 \pm 0.39$ & $78.30 \pm 18.23$ & $77.73 \pm 16.28$ \\
\hline \multirow[t]{2}{*}{ PL } & Video & $.66 \pm .09$ & $.21 \pm .12$ & $1.219 \pm 0.62$ & $0.197 \pm 0.16$ & $77.8 \pm 11.14$ & $81.5 \pm 12.54$ \\
\hline & PLD & $.51 \pm .15$ & $.29 \pm .21$ & $0.579 \pm 0.92$ & $0.264 \pm 0.32$ & $80.7 \pm 11.95$ & $79.3 \pm 14.29$ \\
\hline
\end{tabular}




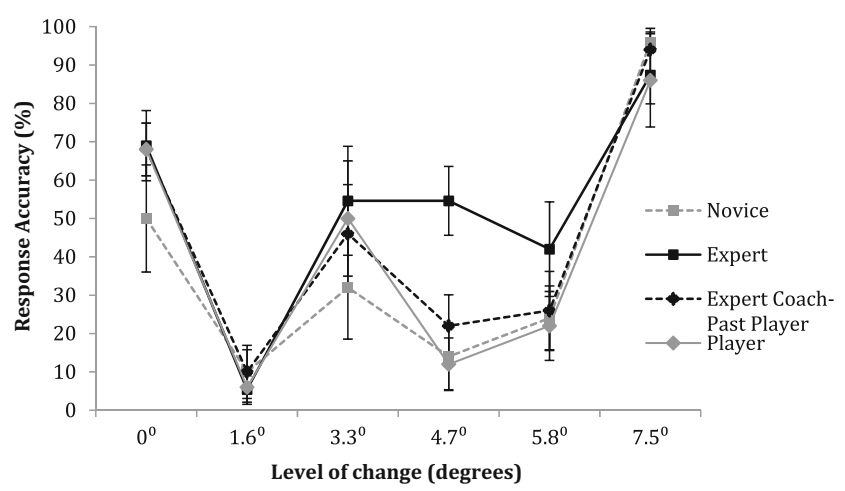

Fig. 3 Response accuracy for all groups in the video condition for knee flexion. Error bars represent the standard errors

novice coaches $(p=.006)$ and players $(p=.044)$ when viewing the video display. A significant two-way interaction between expertise and level of change was also evident in the video viewing condition, $F(15,185)=1.859, p=.030$, $\eta_{\mathrm{p}}{ }^{2}=.131$. Follow-up analyses revealed that the experts significantly outperformed novice coaches $(p<.001)$, expert past players $(p=.003)$, and current players $(p<.001)$ at L4. A significant main effect of level, $F(3.657,135.291)=72.154, p$ $>.001, \eta_{\mathrm{p}}{ }^{2}=.661$, revealed that RA significantly decreased from L1 to L2 $(p<.001)$, significantly increased from L2 to L3 $(p<.001)$, significantly decreased from L3 to L4 ( $p=.013)$, and remained similar between L4 and L5, before significantly increasing again at L6, the largest change $(p<.001)$.

In the PLD condition, we found no significant main effect of expertise, $F(3,37)=1.615, p=.202, \eta_{\mathrm{p}}{ }^{2}=.116$; there was, however, a significant main effect of level, $F(3.605$, $133.388)=78.632, p<.001, \eta_{\mathrm{p}}{ }^{2}=.680$, and a significant Expertise $\times$ Level of Change interaction, $F(15,185)=$ $1.909, p=.025, \eta_{\mathrm{p}}{ }^{2}=.1314$. Follow-up analyses revealed that both experts $(p=.006)$ and players $(p=.019)$ outperformed novices at L1 when viewing the PLD. Pairwise comparisons with Bonferroni correction revealed that RA scores decreased significantly from L1 to L2 $(p<.001)$, remained consistent though L5, before significantly increasing at the largest change in L6 $(p<.001)$. A Spearman correlation revealed

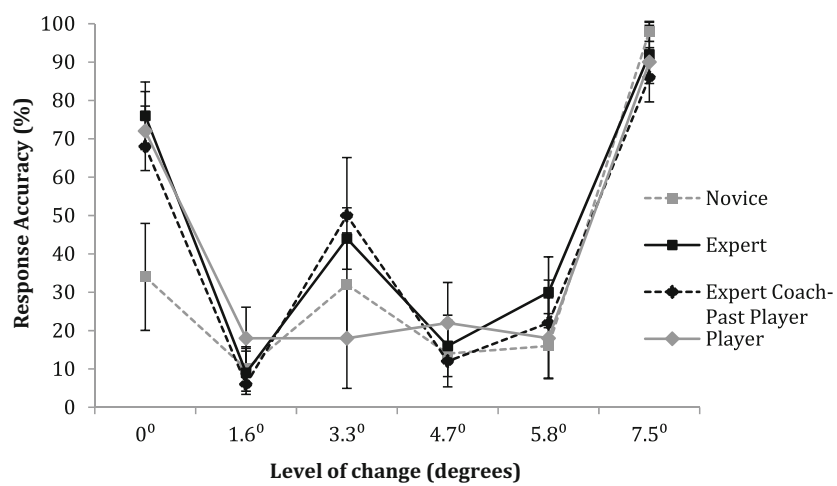

Fig. 4 Response accuracy for all groups in the PLD condition for knee flexion. Error bars represent the standard errors no significant relationship between RA and confidence, $r_{\mathrm{s}}(492)=.069, p=.063$.

Trunk rotation There was no significant effect of expertise on RA after controlling for the effect of age, $F(3,36)=0.296$, $p=.828, \eta_{\mathrm{p}}{ }^{2}=.024$ (see Figs. 5 and 6). No higher-order interactions between coaching expertise, level of change, and viewing perspective, $F(15,185)=0.841, p=.632$, or level of change and viewing perspective, $F(5,185)=1.758, p=.112$, were present. The main effect of level of change was significant, $F(5,185)=24.042, p<.001, \eta_{\mathrm{p}}{ }^{2}=.404$, whereas the main effect of viewing condition was not, $F(1,37)=0.243, p=.625$. Pairwise comparisons with Bonferroni corrections applied revealed that RA scores decreased significantly from L1 to L2 $(p<.05)$ and remained constant from L3 to L5, before significantly decreasing again at L6 $(p<.05)$. A Spearman correlation revealed no significant relationship between RA and confidence, $r_{\mathrm{s}}(492)=-.003 p=.476$.

Ball toss positioning We observed no significant effect of expertise on RA after controlling for the effect of age, $F(3$, $36)=1.409, p=.256, \eta_{\mathrm{p}}{ }^{2}=.105$ (see Figs. 7 and 8). No significant three-way interaction between coaching expertise, level of change, and viewing perspective was evident, $F(10.243,126.324)=0.475, p=.906$; however, there was significant two-way interaction between viewing perspective and level of change, $F(3.414,126.324)=4.326, p=$ $.004, \eta_{\mathrm{p}}^{2}=.105$. In the video viewing condition, a significant difference in RA between levels of change, $F(3.491$, $129.177)=71.322, p<.001, \eta_{\mathrm{p}}{ }^{2}=.658$, revealed that RA decreased significantly from L1 to L2 $(p<.001)$, remained similar from L2 to L3 $(p=.127)$, significantly increased from L3 to L4 $(p<.001)$, and then remained similar for L5 $(p=.701)$ and L6 $(p=.110)$. We also found a significant main effect of level of change on RA in the PLD condition, $F(3.245,120.082)=47.916, p<.001, \eta_{\mathrm{p}}{ }^{2}=.564$. Pairwise comparisons, with Bonferroni corrections, revealed that RA scores decreased significantly from L1 to L2 $(p<.001)$, remained similar at L3 $(p=1.00)$, significantly increased at L4 $(p<.001)$, and remained similar at L5 $(p=.712)$, before significantly increasing at L6, the largest change $(p=.002)$. Overall, a Spearman correlation revealed no significant correlation between confidence and RA, $r_{\mathrm{s}}(492)=.026, p=.285$.

\section{Discussion}

The aim of this study was to determine the effects of coaching and playing experience on the perception of kinematic change. The perceptual capabilities of a sample of expert coaches, novice coaches, expert coaches with high-level playing experience, and current players were tested on two tasks; (1) a global discrimination task that required the coaches to identify 


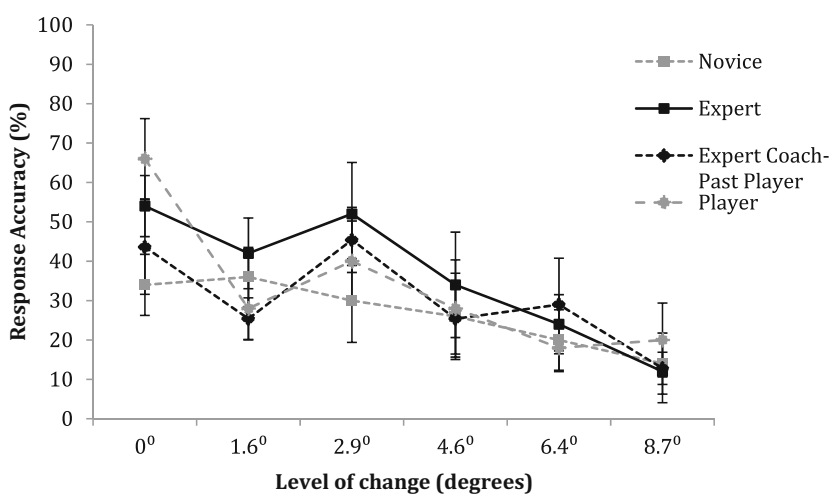

Fig. 5 Response accuracy for all groups in the video condition for trunk rotation. Error bars represent the standard errors

serves as either the same or different, and (2) a specific discrimination task that required the coaches to identify how designated kinematic features within a comparison serve differed from those in a reference serve. Judgments were made from displays (PLDs) that only provided kinematic information, as well as from normal video displays that provided both kinematic and nonkinematic sources of information. It was expected that expert coaches (including those with motor expertise) would show increased sensitivity to perceiving change relative to novice coaches, with the addition of playing (motor) expertise being expected to provide some benefit above and beyond perceptual experience alone.

\section{Global discrimination task findings}

Neither coaching nor playing experience appeared to provide a perceptual advantage above novice levels of perceptual experience when judging global changes in kinematics. All coaches, regardless of their coaching and playing experience, demonstrated similar sensitivities to detecting global changes. Not surprisingly, hit rates decreased while false alarm rates increased when viewing the PLDs as compared to the full videos, highlighting the difficulty faced by coaches when

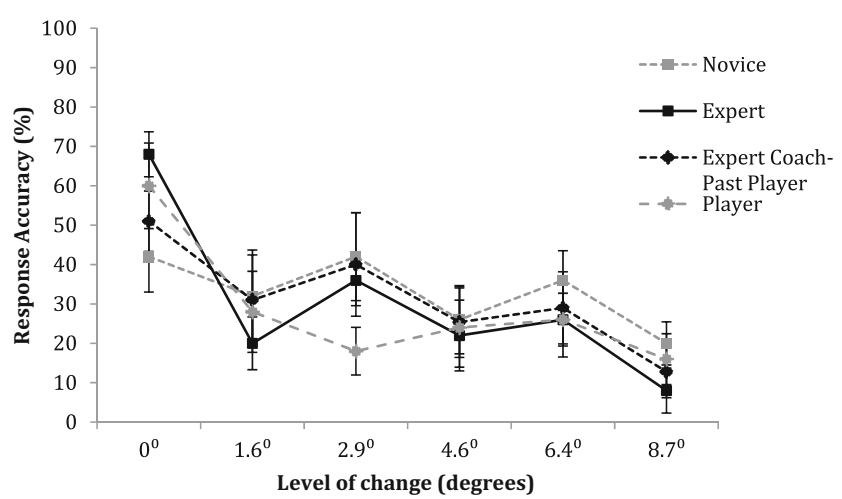

Fig. 6 Response accuracy for all groups in the PLD condition for trunk rotation. Error bars represent the standard errors

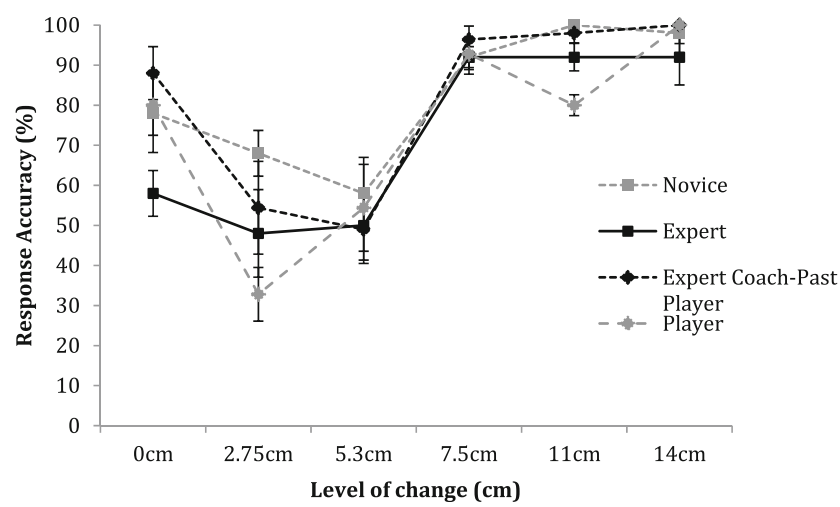

Fig. 7 Response accuracy for all groups in the video condition for ball toss positioning. Error bars represent the standard errors

making a judgment in the absence of contextual information. Interestingly, the criterion increased from video to PLD, suggesting that all coaches were more conservative when viewing the PLDs, and thus less likely to report the signal as different. Given the significant decrease in response accuracy (hit rate) and the decreased sensitivity (lower $d$ prime), the results failed to support either our prediction that expert coaches would show better global discrimination sensitivity or that such judgments would be based primarily on the underlying kinematics of the action.

\section{Specific discrimination task findings}

The results provided some support to our contention that expert coaches would be more sensitive to changes in maximum knee flexion than would either NCs or players. EC1s showed a capacity to detect all changes in the video condition with similar degrees of accuracy, except for the smallest change of $1.6^{\circ}$. A group main effect for expertise highlighted the overall superior performance of EC1s than of NCs and PLs, with EC1s outperforming all other groups at L4 $\left(4.7^{\circ}\right)$. The same pattern of results was not evident when EC1s viewed the PLDs, with significantly poorer

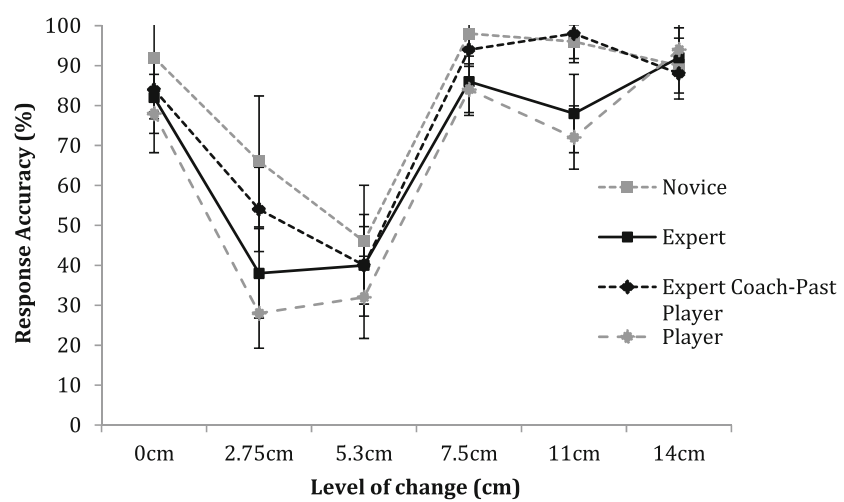

Fig. 8 Response accuracy for all groups in the PLD conditions for ball toss positioning. Error bars represent the standard errors 
performance at both Levels 3 and 4 (where previously performance had been above chance level) suggesting that expert coaches were likely basing their judgments on contextual information available within the video display. NCs performed at below-chance levels when detecting all changes from $0^{\circ}$ to $5.8^{\circ}$, suggesting that changes must be larger than $5.8^{\circ}$ for NCs to detect them reliably. Such a finding is not surprising, given that NCs spend the majority of their time analyzing the service action of beginning and novice players, who typically have large variations in technique. The combination of perceptual and motor expertise did not appear to assist the EC2s. In a similar vein to the $\mathrm{NCs}$, the EC2 coaches performed comparably in both viewing conditions, with RA greatest when the largest angular difference was presented. The PLs performed similar to the EC2s in both viewing conditions, with RA also significantly decreasing when they viewed PLDs at L4. Inspection of Figs. 3 and 4 clearly shows the main difference occurred at Levels 3 and 4 (the $3.3^{\circ}$ and $4.7^{\circ}$ changes), providing evidence suggesting that some stimulus presented within these clips aided the perception of change, one that wasn't available when viewing only the kinematics of the action in the PLD condition.

Knowledge is considered an important aspect of coaching expertise and has been characterized as one of many qualities separating expert from less-skilled coaches (Côté, Salmela, Trudel, \& Baria, 1995; Leas \& Chi, 1993). It is possible that even though coaches initially viewed the knee action with the aim of detecting a change, they also used other available information to reinforce their decision. For instance, the coaches may have used information from the racket-ball contact point to infer greater knee flexion, and therefore greater leg drive that enabled the player to reach a higher contact point. Given that NCs and PLs have had less perceptual experience analyzing the serve, they may not have been able to use or interpret the additional biomechanical information available in the serve as effectively as the EC1s. SteMarie (1999) proposed a similar notion with judges, suggesting that expert judges outperform novices at predicting the upcoming element in a sequence of gymnastic maneuvers because they are better able to understand the biomechanical information available to them. Further support for this proposition can be gained from the work of Abernethy and Russell (1987), who examined the visual search behavior and anticipatory skill of expert and novice badminton players in a series of experiments. Their visual search results suggested that although experts and novices exhibited the same search behavior, the experts revealed a greater capacity to extract and use relevant information from the display.

Changes in trunk kinematics were difficult to perceive for all groups. Despite the trunk area being a major focus for expert coaches when analyzing the serve (Giblin, Farrow, Reid, Ball, \& Abernethy, 2013), no group was able to accurately perceive or use the relevant rotational information to inform their judgment. It is conceivable that when analyzing the serve, experts are using the trunk as a central visual pivot point, but also rely heavily on peripheral vision to obtain information (Rienhoff, Baker, Fischer, Strauss, \& Schorer, 2012; Ryu, Abernethy, Mann, Poolton, \& Gorman, 2013). If this is the case, their priority for viewing the trunk may not necessarily translate into an increased capacity to detect changes in this area. Interestingly, for all groups, RA decreased as the level of change increased, contrary to our hypothesis. It is possible that changes were difficult to detect due to the motion of other joints (e.g., the shoulder) in the area undergoing maximum displacement, which may make direct perception of trunk rotation difficult. Another potential reason this pattern of results could have emerged may be the availability of additional kinematic information contained within each clip. Given the difficulty of detecting change directly from trunk rotation, it is likely that coaches were using other kinematic information to infer the amount of trunk rotation in the serve. For example, if the player landed farther into court during the followthrough, the coach might have used this to infer that greater trunk rotation had occurred, despite not being able to perceive the change directly from the trunk. This may account for the accurate perception on clips in which a specific kinematic difference (but not the intended one) was noticeably perceived. In contrast, working backward to infer a change could just as easily lead to incorrect judgments, and therefore account for the poor performance when perceiving the larger changes in trunk rotation.

Coaching experience did not seem to benefit the EC1s or EC2s when perceiving changes in ball toss positioning. In fact, all groups easily perceived changes in ball toss positioning at above-chance levels, even in the absence of contextual information. When analyzing the serve, novices have been shown to spend significantly more time viewing the ball than do expert coaches (Giblin et al., 2013). In this instance, it is possible that the level of perceptual skill attained by NCs guided their gaze behavior, rather than letting the importance of the underlying kinematics guide their visual search. That is, NCs are most competent in detecting changes in ball flight characteristics, and as a result spend the majority of their time viewing the ball. These results may be comparable to the temporal occlusion research in the performance literature, which has demonstrated that less-skilled players are more reliant on ball flight information in anticipation tasks, whereas experts possess the skill to interpret and use advance kinematic information, which novices are unable to interpret (Abernethy, 1990; Farrow et al., 2005). 
In terms of confidence, all coaches were highly confident in their judgments for both the global and specific tasks. In most instances, the coaches' self-reported confidence in their responses was well above their objective RA. This is confirmed by the poor correlations between RA and confidence, which were unexpected. Given that the role of the coach is to guide development and feedback, coaches need to convey a certain conviction in their feedback, which may account for what might be perceived as misplaced or overconfidence. Given that a potential problem when examining expertise is the inability of experts to accurately self-report the source of their expertise (Abernethy, 1994), the use of subjective thresholds (the point at which coaches believe they are guessing, but in fact they are still responding at above-chance levels) may allow future research to consider the degree of change that an observer can consciously detect as well as the extent of change to which they can (often unconsciously) respond.

\section{Conclusions and implications}

Although we did not seek to directly examine the commoncoding hypothesis, our findings raise some interesting questions with regard to motor expertise. Although they are not directly comparable, given the different tasks used (anticipation vs. discrimination) and likely differences in group expertise characteristics, our results contrast with those from a number of studies that have examined expertise in anticipation. In those studies (Aglioti et al., 2008; Cañal-Bruland, van der Kamp, \& van Kesteren, 2010), motor experience was deemed to facilitate anticipation above perceptual experience alone. One possible reason we did not find the same pattern of results relates to the task used. Although the tasks were fundamentally similar, in that both anticipation and kinematic discrimination tasks require the pickup of informative kinematics, we hypothesize that performance on the discrimination task may have also been influenced by cognitive factors, particularly knowledge structures, given their known contributions to expertise in both playing (McPherson, 1993; McPherson \& Thomas, 1989) and coaching (Leas \& Chi, 1993). Some support for this argument can be gained from the superior performance of the EC1s in the knee discrimination task (particularly at Level 4), a result that was likely due to a combination of their increased experience and higher formal training or knowledge. Given this, it seems that increased experience and knowledge may have allowed the EC1s to extract and use additional information contained in this specific clip, as compared to NCs, to reinforce or inform their judgments. Given that extensive knowledge is considered a primary characteristic of coaching expertise (Leas \& Chi, 1993; Schempp, McCullick, \& Mason, 2006), it seems logical to conclude that experience, and through this knowledge, is a critical factor in the development of the perceptual skill to discern and discriminate changes in movement kinematics.
A limitation of the present study pertains to the difficulty of experimentally separating experience from expertise. It is possible that playing experience, and not necessarily motor expertise, is enough to facilitate the perception of change, which may explain the lack of expertise effects observed in the present study. Furthermore, whereas we recruited all available coaches who met the criteria for each expertise group, the limited number of coaches who had the qualifications and experience to actually meet the criteria (particular for the EC2 group) meant that the study was potentially underpowered. Furthermore, differences in age between participants potentially confounded the results. Although experimentally controlling for age is difficult, given the nature of the task, age was considered as a covariate and did not significantly influence the study outcomes.

A secondary hypothesis, that the perception of motion is based on the underlying kinematics of the action, was tested through the use of PLDs. Our findings provide some support to previous research using anticipation tasks (Ward, Williams, \& Bennett, 2002) that had demonstrated that expertise differences are maintained when viewing PLDs, despite a decrement in overall performance levels. When holistically discriminating changes in technique, no expertise effects were evident, but viewing condition did significantly influence RA. Despite a decrease in RA when viewing the PLDs, the same pattern of results with regard to RA and expertise was not evident in the PLD condition, suggesting that global judgments of movement pattern change were not based primarily on the kinematics of the action. In contrast, when discriminating specific changes in kinematics, the same broad pattern of RA results was observed in the video and PLD conditions, albeit often with significantly decreased accuracy in the PLD condition, suggesting that the discrimination of change in specific features of the service action is likely based on the underlying kinematics of the action.

Given the dynamic nature of the tennis serve, direct measurement of perceptual sensitivity to change is difficult. Although we attempted to examine perceptual sensitivity to specific kinematic changes, given the degrees of freedom involved in the serve, it is impossible to prevent other kinematics from changing at the same time. As we previously mentioned, it is possible that the coaches used these additional changes to reinforce or infer their decisions, making direct conclusions regarding the perception of specific kinematics difficult. The addition of eye tracking in future studies will be necessary to help establish where coaches direct their attention during the performance of such a discrimination task. Even though in the present study the participants were directed to attend to each specific kinematic variable, it would be interesting to establish whether coaches do indeed fixate on these variables and, in the event that they can't detect a change directly, it would be valuable to know what other sources of information they may seek to use to infer or confirm their 
decisions. Given that previous research on the dynamic information that underpins anticipation performance has suggested that skilled performers adopt a more "global" rather than a "local" strategy, relying on multiple sources of information to inform their judgments (Williams, Huys, CañalBruland, \& Hagemann, 2009), it would be interesting to uncover whether skilled coaches take a similar "global" approach.

Typically, research examining perceptual sensitivity has occurred in controlled clinical settings rather than more dynamic environments such as those in sport. In the present study, we attempted to adapt the JND method, common in psychophysics research, to a highly complex and coordinated movement. Although the degree of change presented to the coaches was incrementally increased, the direction (increasing or decreasing angular position) changed. It is possible that in the present study, changes in movement direction confounded the results. For example, if increases in angular position are easier to perceive, an increase of $3^{\circ}$ might be perceived, whereas a decrease of $3^{\circ}$ might not. If this is the case, a clear point at which changes in kinematics are perceptible becomes difficult to measure. Future research should more systematically consider movement direction, as well as movement plane, since the present findings suggest that movements in the transverse plane (in this case, trunk rotation) may be more difficult to perceive than movements in the sagittal plane (knee flexion).

Although the present study suggests that coaches are quite sensitive to small changes in knee flexion and ball toss positioning, it will be critical to consider the importance or impact of these changes. Movement production is inherently variable, with small changes in kinematics often being present despite successful skill execution. From an ecological-dynamics perspective, movement variability helps an individual adapt to unique constraints (Davids, Glazier, Araujo, \& Bartlett, 2003) and facilitates flexible coordination tailored to specific environmental or task demands (Kelso, 1997). As a coach, it is crucial to consider whether changes in kinematics are functionally variable or whether significant errors are likely to affect skill execution. Increased sensitivity to changes in movement kinematics is likely to be redundant if a coach is unable to perceive the difference between inherent variability and authentic skill errors. Furthermore, it is also critical to consider the corresponding sensitivity of performers to implement kinematic changes in their movement patterns. For instance, if visual sensitivity is superior to the performer's kinesthetic sensitivity, any feedback provided at the most precise level of visual sensitivity will be beyond the level that performers can use effectively. For this reason, it will be critically important in future studies to also consider the visual system and the kinesthetic system together when assessing the likely efficacy of feedback provided by coaches.

\section{References}

Abernethy, B. (1990). Anticipation in squash: Differences in advance cue utilization between expert and novice players. Journal of Sports Sciences, 8, 17-34. doi:10.1080/02640419008732128

Abernethy, B. (1994). The nature of expertise in sport. In S. Serpa, J. Alves, \& V. Pataco (Eds.), International perspectives on sport and exercise psychology (pp. 57-68). Morgantown, PA: Fitness Information Technology.

Abernethy, B., Gill, D. P., Parks, S. L., \& Packer, S. T. (2001). Expertise and the perception of kinematic and situational probability information. Perception, 30, 233-252.

Abernethy, B., \& Russell, D. G. (1987). The relationship between expertise and visual search strategy in a racquet sport. Human Movement Science, 6, 283-319.

Abernethy, B., \& Zawi, K. (2007). Pickup of essential kinematics underpins expert perception of movement patterns. Journal of Motor Behavior, 39, 353-367.

Aglioti, S. M., Cesari, P., Romani, M., \& Urgesi, C. (2008). Action anticipation and motor resonance in elite basketball players. Nature Neuroscience, 11, 1109-1116.

Buccino, G., Binkofski, F., \& Riggio, L. (2004). The mirror neuron system and action recognition. Brain and Language, 89, 370-376.

Calvo-Merino, B., Glaser, D. E., Grèzes, J., Passingham, R. E., \& Haggard, P. (2006). Action observation and acquired motor skills: An FMRI study with expert dancers. Cerebral Cortex, 15, 12431249.

Cañal-Bruland, R., van der Kamp, J., \& van Kesteren, J. (2010). An examination of motor and perceptual contributions to the recognition of deception from others' actions. Human Movement Science, 29, 94-102.

Coté, J., \& Gilbert, W. (2009). An integrative definition of coaching effectiveness and expertise. International Journal of Sports Science \& Coaching, 4, 307-323.

Coté, J., Salmela, J., Trudel, P., \& Baria, A. (1995). The coaching model: A grounded assessment of expert gymnastic coaches' knowledge. Journal of Sport \& Exercise Psychology, 17, 1-17.

Davids, K., Glazier, P., Araujo, D., \& Bartlett, R. (2003). Movement systems as dynamical systems. Sports Medicine, 33, 245-260.

Elliott, B., Reid, M., \& Crespo, M. (2003). ITF biomechanics of advanced tennis. Roehampton, UK: International Tennis Federation.

Ericsson, K. A., Krampe, R. T., \& Tesch-Römer, C. (1993). The role of deliberate practice in acquisition of expert performance. Psychological Review, 100, 363-406. doi:10.1037/0033-295 X.100.2.363

Farrow, D., \& Abernethy, B. (2003). Do expertise and the degree of perception-action coupling affect natural anticipatory performance? Perception, 32, 1127-1140.

Farrow, D., Abernethy, B., \& Jackson, R. C. (2005). Probing expert anticipation with the temporal occlusion paradigm: Experimental investigations of some methodological issues. Motor Control, 9, 330-349.

Ford, P. R., Coughlan, E., \& Williams, M. (2009). The expertperformance approach as a framework for understanding and enhancing coaching performance, expertise and learning. International Journal of Sports Science \& Coaching, 4, 451-463. doi:10.1260/174795409789623919

Giblin, G. L., Farrow, D., Reid, M., Ball, K., \& Abernethy, B. (2013). Keep your eye off the ball: Expertise differences in visual search behavior of tennis coaches [Abstract]. Journal of Sport \& Exercise Psychology, 35(Supp), S29.

Gründel, A., Schorer, J., Strauss, B., \& Baker, J. (2013). Does playing experience improve coaching? An exploratory study of perceptualcognitive skill in soccer coaches. Frontiers in Psychology, 4, 129. doi:10.3389/fpsyg.2013.00129 
Kantowitz, B. H., Roediger, H. L., \& Elmes, D. G. (2009). Experimental psychology: Understanding psychological research. Sydney, Australia: Wadsworth Thomson Learning.

Kelso, J. (1997). Relative timing in brain and behavior: Some observations about the generalized motor program and self-organized coordination dynamics. Human Movement Science, 16, 453-460.

Leas, R. R., \& Chi, M. T. H. (1993). Analyzing diagnostic expertise of competitive swimming coaches. In J Starkes \& F. Allard (Eds.), Advances in psychology: Cognitive issues in motor expertise (Vol. 102, pp. 75-94). Amsterdam, The Netherlands: North-Holland.

Lloyd, D., Alderson, J., \& Elliott, B. (2000). An upper limb kinematic model for the examination of cricket bowling: A case study of Mutiah Muralitharan. Journal of Sports Sciences, 18, 975-982.

Macmillan, N. A., \& Creelman, C. D. (2004). Detection theory: A user's guide (2nd ed.). Mahwah, NJ: Erlbaum.

McPherson, S. L. (1993). The influence of player experience on problem solving during batting preparation in baseball. Journal of Sport \& Exercise Psychology, 15, 304-325.

McPherson, S. L., \& Thomas, J. R. (1989). Relation of knowledge and performance in boys' tennis: Age and expertise. Journal of Experimental Child Psychology, 48, 190-211.

Müller, S., Abernethy, B., \& Farrow, D. (2006). How do world-class cricket batsmen anticipate a bowler's intention? Quarterly Journal of Experimental Psychology, 59, 2162-2186.

Pizzera, A. (2012). Gymnastic judges benefit from their own motor experience as gymnasts. Research Quarterly for Exercise and Sport, $83,603-607$.

Prinz, W. (1997). Perception and action planning. European Journal of Cognitive Psychology, 9, 129-154.

Reid, M., Whiteside, D., Giblin, G., \& Elliott, B. (2013). Effect of a common task constraint on the body, racket, and ball kinematics of the elite junior tennis serve. Sports Biomechanics, 12, 15-22.

Rienhoff, R., Baker, J., Fischer, L., Strauss, B., \& Schorer, J. (2012). Field of vision influences sensory-motor control of skilled and less- skilled dart players. Journal of Sports Science and Medicine, 11, 542-550.

Ryu, D., Abernethy, B., Mann, D. L., Poolton, J. M., \& Gorman, A. D. (2013). The role of central and peripheral vision in expert decision making. Perception, 42, 591-607.

Schempp, P., McCullick, B., \& Mason, I. (2006). The development of expert coaching. In R. Jones (Ed.), The sports coach as educator: Reconceptualising sports coaching (pp. 145-161). London, UK: Routledge.

Schütz-Bosbach, S., \& Prinz, W. (2007). Perceptual resonance: Actioninduced modulation of perception. Trends in Cognitive Sciences, 11, 349-355.

Sherman, C. A., Sparrow, W., Jolley, D., \& Eldering, J. (2001). Coaches' perceptions of golf swing kinematics. International Journal of Sport Psychology, 31, 257-270.

Ste-Marie, D. M. (1999). Expert-novice differences in gymnastic judging: an information processing perspective. Applied Cognitive Psychology, 13(3), 269-281.

Ward, P., Williams, A. M., \& Bennett, S. J. (2002). Visual search and biological motion perception in tennis. Research Quarterly for Exercise and Sport, 73, 107-112.

Weir, P., Holmes, A., Andrews, D., Albert, W., Azar, N., \& Callaghan, J. (2007). Determination of the just noticeable difference (JND) in trunk posture perception. Theoretical Issues in Ergonomics Science, 8, 185-199.

Whiteside, D., Chin, A., \& Middleton, K. (2013). The validation of a three-dimensional ball rotation model. Proceedings of the Institution of Mechanical Engineers, Part P: Journal of Sports Engineering and Technology, 227, 49-56.

Williams, M., Huys, R., Cañal-Bruland, R., \& Hagemann, N. (2009). The dynamical information underpinning anticipation skill. Human Movement Science, 28, 362-370.

Wiman, M., Salmoni, A. W., \& Hall, C. R. (2010). An examination of the definition and development of expert coaching. International Journal of Coaching Science, 4, 37-60. 